reasonable, then condition $\left(9.18^{\prime \prime}\right)$ is more stringent than $\left(9.18^{\prime}\right)$ and we therefore regard it as the critical condition regarding the relative precision of the approximate inverse.

Considering the precisions (7.17.a)-(17.17c.), we find that $\left(9.18^{\prime \prime}\right)$ becomes

$$
\begin{aligned}
& n<19 \\
& n<86, \\
& n<400
\end{aligned}
$$

InSTITUTE For Advanced StUdy

\title{
ON SERIES OF WALSH EIGENFUNCTIONS
}

W. H. SPRAGENS

Walsh $[4]^{1}$ considered expansions in terms of eigenfunctions satisfying the equation

$$
u^{\prime \prime}(x)+\left[\rho^{2}-g(x)\right] u(x)=0, \quad 0 \leqq x \leqq 1,
$$

and the boundary conditions

$$
u(0)=0, \quad u(1)=0,
$$

the function $g(x)$ being assumed continuous on $0 \leqq x \leqq 1$. He used the asymptotic formula for the $k$ th eigenfunction

$$
\bar{u}_{k}(x)=(2)^{1 / 2}\left[\sin k \pi x+(1 / k) \phi_{k}(x)\right], \quad\left|\phi_{k}(x)\right| \leqq C .
$$

Comparing series of these functions with corresponding series of the functions

$$
u_{k}(x)=(2)^{1 / 2} \sin k \pi x,
$$

he proved that if a function in $L^{2}$ is expanded in terms of both sets of functions, then the series of term-by-term differences converges uniformly and absolutely to zero on $0 \leqq x \leqq 1$.

This result, as Walsh points out, is closely related to that of Haar [1], who considered the Sturm-Liouville problem with boundary conditions $u^{\prime}+H u=0$, and compared the series of the resulting functions with the Fourier cosine series. He obtained equiconvergence in

Received by the editors April 3, 1950.

${ }^{1}$ Numbers in brackets refer to the references cited at the end of the paper. 
the sense that the sequence of differences of partial sums approaches zero. While this is weaker than the absolute equiconvergence of Walsh's theorem, Haar's result is valid for expansions of a broader class of functions, namely, all Lebesgue-integrable functions.

We show here that a result of equal scope may be deduced for Walsh's problem, if the method of Haar is used; for the latter method depends principally on the boundedness of the "difference-kernel," which may be shown readily.

THEOREM. If a Lebesgue-integrable function on the interval $0 \leqq x \leqq 1$ be expanded in terms of Walsh's functions (1) and the sine functions (2), then the two series are uniformly equiconvergent on the interval.

The Dirichlet kernel for the Walsh functions is

$$
\bar{D}_{n}(x, s)=\sum_{k=1}^{n} \bar{u}_{k}(x) \bar{u}_{k}(s) .
$$

By using the asymptotic formula (1) we see that the difference between this kernel and the corresponding kernel for the sine functions (2) is of the form

$$
\begin{aligned}
\Phi_{n}(x, s) & \equiv \bar{D}_{n}(x, s)-D_{n}(x, s) \\
& =2 \sum_{k=1}^{n}\left(\frac{\phi_{k}(x) \sin k \pi s}{k}+\frac{\phi_{k}(s) \sin k \pi x}{k}+\frac{\phi_{k}(x) \phi_{k}(s)}{k^{2}}\right) .
\end{aligned}
$$

This difference-kernel is readily seen to be bounded independently of $n, x$, and $s$.

Now we use the Riemann-Lebesgue theorem in the following form given by McShane [2, p. 231]: that

If $I$ is a closed interval and $\left\{g_{n}(x)\right\}$ a sequence of functions such

(i) all the $g_{n}(x)$ have the same bound on $I$;

(ii) each $g_{n}(x)$ is measurable on $I$;

(iii) on each subinterval $I_{0}$ of $I$,

$$
\lim _{n \rightarrow \infty} \int_{I_{0}} g_{n}(x) d x=0 ;
$$

then for every $f(x)$ summable over $I$, and for each subinterval $I_{0}$ of $I$,

$$
\lim _{n \rightarrow \infty} \int_{I_{0}} f(x) g_{n}(x) d x=0 .
$$

We use for $g_{n}(s)$ in this theorem the difference-kernel $\Phi_{n}(x, s), x$ 
being a fixed point on the interval $I:[0,1]$. Condition (iii) follows from Walsh's result applied to the characteristic function of the subinterval $I_{0}$, this function being of integrable square. So we have for any function $f(x)$ summable over $I$

$$
\lim _{n \rightarrow \infty} \int_{I} f(s) \Phi_{n}(x, s) d s=0,
$$

this limit, moreover, being approached uniformly with respect to $x$ on account of the corresponding uniformity in conditions (i) and (iii). The theorem follows.

The results of Haar [1] were extended to double series by Mitchell [3]. We note here that the theorems on equiconvergence and equisummability between the double Fourier cosine series and the double Sturm-Liouville (Haar) series which she obtains hold likewise between the double sine series and the double Walsh series. Her proofs go through practically unchanged; the formulae for the kernels of the cosine series and of the sine series are alike except for a sign which does not affect the argument, and the difference-kernels, as mentioned previously, are bounded in both cases.

\section{REFERENCES}

1. A. Haar, Zur theorie der orthogonalen Funktionensysteme, Math. Ann. vol. 69 (1910) pp. 331-371.

2. E. J. McShane, Integration, Princeton, 1944.

3. Josephine Mitchell, On double Sturm-Liouville series, Amer. J. Math. vol. 65 (1943) pp. 616-636.

4. J. L. Walsh, On the convergence of Sturm-Liouville series, Ann. of Math. vol. 24 (1922) pp. 109-120.

UNIVERSITY OF MISSISSIPPI 